
Military Technical College
Kobry El-Kobbah,
Cairo, Egypt.

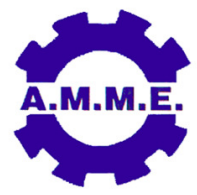
$16^{\text {th }}$ International Conference on Applied Mechanics and Mechanical Engineering.

\title{
A MICROMECHANICS-BASED DAMAGE DIAGNOSTIC MODEL FOR MATERIALS
}

\author{
M. E. Abdel-Meguid", K. Shalan", T. M. Hatem ${ }^{* *}$ and Y. A. Bahei El-Din ${ }^{* *}$
}

\begin{abstract}
The complexity of engineering structures dictates a paradigm shift from traditional inspection and damage detection techniques to more reliable and efficient approach. Smarts materials such as piezoelectric materials are being studied as onboard sensors to detect damage progression inside composite structures. Nevertheless, predictive models of such complex structures coupled to piezoelectric materials been absent, especially related to damage detection and prediction. In the current study, a multi-scale approach is suggested to predict the behavior of piezoelectric fiber-based composites. Micromechanical model based on transformation field analysis is described to quantify the overall material properties of electrically active composite structure. Capitalizing on the extracted properties, single-phase analysis of a homogeneous structure is conducted using Carrera Unified formulation; a refined plate theory extended to include electric behavior of active materials. Results obtained here are validated against experimental results. Furthermore, the impact of damage on local and global fields is evaluated on macro-level through simulated voids inside a beam-like structure.
\end{abstract}

\section{KEY WORDS}

Piezoelectric, composites, and damage identification.

* Graduate student, Center for Advanced Materials, the British University in Egypt, El-Shorouk City, Egypt

** Associate Professor, Center for Advanced Materials, the British University in Egypt, El-Shorouk City, Egypt

*** Professor, Center for Advanced Materials, the British University in Egypt, ElShorouk City, Egypt 


\section{INTRODUCTION}

Early failure in engineering structure became a concern with the increased employment of composite structures and the complexity of its applications. Conventional inspection techniques are no longer reliable to assess the integrity of these complex structures, especially composite-based structures. The use of composites has been substantially enhancing the mechanical properties of engineering structures; however, their nature still increases the susceptibility of the structure to incipient failure. Accordingly, continuous real-time health monitoring systems are being considered to replace conventional inspection techniques, mainly, to actively identify damage progression inside these complex structures. Several studies identified the role of adaptive smart materials in structural health monitoring applications; embedded smart materials that are responsive toward external stimuli can be actively utilized as sensors or actuators inside structures [1]. Electrically active materials, that couple mechanical and electrical properties such as piezoelectric material, became a top candidate in damage identification applications. Initial investigations focused on the applicability of piezoelectric wafers embedded within the composite laminates [2-5]. However, with recent advancements in manufacturing technology, the focus shifted towards the behavior of polymer-based composites with embedded electrically active Lead Zirconate Titnate fibers [PZT] [68].

Numerical modeling of piezoelectric fiber-based composites [PFC] is mainly focused on interpretations of overall behavior of composite-structures subjected to electric or mechanical actuation. Analytical quantification of effective properties in active composites was subject to rigorous scrutiny. However, only few studies described the impact of damage on the coupled behavior of PFCs been published. The spectrum of solutions suggested for composites with active constituents are based on expanded problems for inactive composite material, which include electromechanical coupled effect. Earlier models computed effective electro-mechanical properties of a composite material based on the Eshelby proposed solution for an infinite matrix with ellipsoidal inclusion [9-12]. A different methodology involves asymptotic expansion homogenization techniques to extrapolate the electromechanical behavior of electrically active composite materials. Imposing mechanical and non-mechanical boundary conditions, a unit cell model can be used to characterize an idealized periodic geometry through the governing equations of local fields [13]. Bahei-El-Din further expanded on this approach to compute the overall electro-mechanical response of electrically active woven composites using transformation field analysis while accounting for damage progression in composite lamina [14].

On the other hand, Carrera [15-16] developed a computational method to conduct beam analysis on piezoelectric ceramics for actuating and sensing purposes using advanced plates and shells theories. Expanding beam theories to incorporate the electrical response associated with piezoelectricity, Carrera Unified Formulation [CUF] enhances the result's precision through higher order thickness expansions. 


\section{METHOD}

The suggested computational approach discussed in this paper follow a multi-scale approach to model the electric and mechanical behavior of electrical-active-fiber embedded in a polymeric-matrix. The two stage approach consist of numerical quantification of overall electro-elastic coefficients using Transformation Field Analysis (TFA) scheme and static structural analysis using Carrera Unified Formulation (CUF) to model the impact of damage on electrical response of PFCs. Electrically induced strains in active medium is treated as transformation strains and resultant local fields and overall composite response is quantified through the TFA originally described by Dovark, G. J.[17]; TFA computes the local fields using microgeometry dependent concentrations factors. Micro-properties of composite are used to assemble the stiffness matrices in the governing equations using CUF and variable fields are solved using in-house developed finite element software.

\section{Constitutive Equations}

Piezoelectric response can be defined through two reversible effects; first, the production of an electric field as a direct response to an applied stress; and second, a converse effect represented by mechanical deformation as a response to an applied electric potential. The linearly formulated constitutive equations binding the response of piezoelectric material are standardized by ${ }^{[18]}$. The constitutive equations for piezoelectric material are derived from the thermo-dynamical principles correlating the electric field $[E]$, strain $[S]$, stress $[T]$ and electrical displacement $[D][16,19]$.

$$
\begin{aligned}
& S=s^{E} T+d E \\
& D=d^{T} T+\varepsilon E_{k}
\end{aligned}
$$

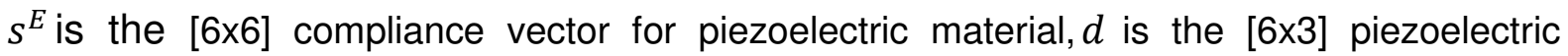
coupling co-efficient vector, $\varepsilon$ is the [3x3] permittivity vector. The two equations can be symmetrically combined in matrix notation.

$$
\left(\begin{array}{l}
S \\
D
\end{array}\right)=\left[\begin{array}{cc}
S & d \\
d^{T} & \varepsilon
\end{array}\right]\left(\begin{array}{l}
T \\
E
\end{array}\right)
$$

Rearranging the constitutive equations for stress function

$$
T=C^{E} S-e E
$$

where, $C^{E}$ is the [6x6] stiffness matrix

$$
e=C^{E} d
$$

For transversely isotropic piezoelectric material, the coefficients can be expressed in matrix notation as the following: 


$$
\begin{gathered}
s^{E}=\left[\begin{array}{cccccc}
s_{11} & s_{12} & s_{13} & 0 & 0 & 0 \\
s_{21} & s_{22} & s_{23} & 0 & 0 & 0 \\
s_{31} & s_{32} & s_{33} & 0 & 0 & 0 \\
0 & 0 & 0 & s_{44} & 0 & 0 \\
0 & 0 & 0 & 0 & s_{55} & 0 \\
0 & 0 & 0 & 0 & 0 & s_{66}
\end{array}\right], \\
d=\left[\begin{array}{cccccc}
0 & 0 & 0 & 0 & d_{15} & 0 \\
0 & 0 & 0 & d_{15} & 0 & 0 \\
d_{31} & d_{31} & d_{33} & 0 & 0 & 0
\end{array}\right], \\
\varepsilon=\left[\begin{array}{ccc}
\varepsilon_{11} & 0 & 0 \\
0 & \varepsilon_{11} & 0 \\
0 & 0 & \varepsilon_{33}
\end{array}\right] .
\end{gathered}
$$

Piezoelectric fiber-based composites consist of transversely isotropic ceramic piezoelectric fibers aligned in an isotropic polymer based matrix. The resultant composite material will be transversely isotropic material represented by 11 mutually exclusive coefficients representing the electro-mechanical response of the homogenized composite.

\section{Transformation Field Analysis (TFA)}

The micromechanical analysis is conducted using the TFA scheme [17] to quantify the overall effective electro-mechanical properties of active PFC composites. Transformation field analysis represents overall response of the material through separating elastic and inelastic fields. All non-mechanical fields due to thermal or electrical actuation are considered as inelastic and treated as transformation fields, which remain in material after removing mechanical loads. The accuracy of the twophase representation is affected by large variations in the transformation field, thus further subdivision for constituents is conducted through a Representative Volume Element [RVE]. The RVE demonstrated in Fig. 1 represents a single cell repeated throughout the material, which under an idealized assumption can provide periodic arrangement. The unit cell used in this transformation field analysis is Periodic Hexagonal Array [PHA][20].

Eigen stresses $[\lambda]$ and strains $[\mu]$ are used to describe transformation fields for the representative volume element. Accordingly the constitutive equations for each element in an RVE can be written to incorporate induced transformation fields due to uniform stress $\left[T_{i}\right]$ or strain $\left[S_{i}\right]$.

$$
\begin{aligned}
& S_{i}=s_{i}^{E} T_{i}+\mu_{i} \\
& T_{i}=C_{i}^{E} S_{i}+\lambda_{i}
\end{aligned}
$$

where $i$ represent number of elements inside the RVE [1,2..Q]. Comparing equations with constitutive equations for PFC, the transformation fields can be attributed to an applied electric field in electrically active piezoelectric fiber-based composites.

$$
\mu_{i}=d_{i} E_{i}
$$




$$
\lambda_{i}=-e_{i} E_{i}
$$

For the RVE entity, the strains and stress caused by uniform stress or strain are super-positioned across the entire volume.

$$
\begin{aligned}
& S_{i}=A_{i} S+\sum_{j=1, \Omega} D_{i j} \mu_{j} \\
& T_{i}=B_{i} T+\sum_{j=1, \Omega} F_{i j} \lambda_{j}
\end{aligned}
$$

where, $\Omega$ is the number of elements carrying transformation fields inside the RVE, and $A_{i}$ and $B_{i}$ are concentration factors used to describe the volume strain and stress in terms of overall counterparts. $D_{i j}$ and $F_{i j}$ are constant influence functions depends mainly on the elastic moduli of each element. These matrices are numerically attained through finite element analysis of the RVE under unit load and respective boundary conditions.

\section{Carrera Unified Formulation}

With overall properties extracted from the transformation field analysis, the extended plate theory can be used to describe the behavior of idealized composite layer. The governing equations for piezoelectric material using PVD can be described with two variables, displacement and electric potential [16].

$$
\begin{gathered}
K_{u u} u+K_{u \phi} \phi=p_{u}-M_{u u} \ddot{u} \\
K_{\phi u} u+K_{\phi \phi} \phi=0
\end{gathered}
$$

where $u, \phi$ stand for the displacement vector and electric potential respectively. While the matrices $\mathrm{K}$ are functions of the electrical and mechanical properties of the material, for multiple layers the matrices are assembled accordingly. $M_{u u}, \ddot{u}$ stand for the inertia matrix and the second derivative of the displacement respectively, and $p_{u}$ signifies the mechanical load exerted.

The variables can be presented by using the unified formation as function of $u(x, y, z)$ and expansion functions in the $z$ direction.

$$
\begin{gathered}
u(x, y, \phi)=\left[\begin{array}{l}
u_{x} \\
u_{y} \\
\phi
\end{array}\right] \\
u(x, y, z, \phi)=\sum_{\tau=1}^{Q} F(z) u(x, y, \phi) \quad \tau=1,2, \ldots . Q
\end{gathered}
$$

The expansion functions are used to achieve higher order approximation across the thickness direction $\tau$ signifies the order of the expansion in the $z$ direction ranging from 1 to $Q$, however, in the formulation the equation can be expanded up to the fourth order. Legrendre polynomials are used to express the expansion functions; their value depends on the element shape and natural coordinates. 
The computation of the variables is done through a finite element model. Shape functions are used to define the relation between the nodal values and the element variable. Accordingly the equation is written as following;

$$
u(x, y, z, \phi)=N_{i} \sum_{\tau=1}^{Q} F(z) q_{i}(x, y, \phi)
$$

In the above equation the $q$ describe the nodal value of the variable vector. Using the above relation the governing equation can be rearranged:

$$
\begin{gathered}
K_{u u}{ }^{i j k \tau s} u^{k}+K_{u \phi}{ }^{i j k \tau s} \phi^{k}=p_{u}-M_{u u} \ddot{u} \\
K_{\phi u}{ }^{i j k \tau s} u^{k}+K_{\phi \phi}{ }^{i j k \tau s} \phi^{k}=0
\end{gathered}
$$

The subscripts $i, j, \tau, s$ are the indices used in the assembly of the stiffness matrix, where $i, j$ and $\tau, s$ are related to the shape functions and expansion functions respectively. Subscript $k$ indicates layer wise analysis for laminated plate structures.

\section{RESULTS}

\section{Pristine Structure}

The multi-scale model applied in the current study is evaluated against experimental data obtained from a manufacturer of PFCs \{Advanced Ceramics Inc\}. The single ply, unidirectional composite consists of PZT-5A fibers and epoxy matrix with 0.45 fiber content. In the RVE the analysis proceeds with applying directional electric field in fiber direction and accordingly the overall composite mechanical deformation is computed at different voltages as shown in Fig. 2. Furthermore, the composite mechanical and electrical properties are extracted for macro-scale analysis using CUF.

Structural analysis using CUF is conducted on the same composite material to verify the modeling approach. The displacement is computed at different applied voltages and compared with experimental results. The mesh geometry shown in Fig. 3a is based on a mesh convergence analysis for the model for accurate representation, while the dimensions are based on the manufacturer datasheet and shown in Fig. 4a. As shown in Fig. 5, the CUF electrically extended formulation, which is based on the constitutive equations for piezoelectric material, follows the same linearized pattern.

\section{Damaged Structure}

Damage is introduced throughout the structure by introducing an elongated (in the ydirection) and thorough (through thickness $-z$ direction) void inside the model in different locations across the composite structure. For constant applied uniaxial load in $y$-direction Fig. 6 represents the adjusted voltage distribution due to void interference. The induced void inside the model caused a global voltage increase from pristine condition across the two electrodes ends. 
The impact of void location is studied through analyses of local voltage fluctuations. For different void locations the local voltage variation is plotted in Fig. 7, the result indicates varying voltage variations.

\section{DISCUSSION}

As shown in Fig. 2, the TFA results present purely linear behavior based on the constitutive equations for piezoelectric material, while the experimental behavior demonstrate a non-linear behavior. The voltage is applied along the fiber direction $\left[x_{3}\right]$ and the displacement is measured in the same direction for alternating voltages. Both the TFA model and experimental results match well on the initial linear part of the curve with low voltage applied, yet at very high voltages the experimental data starts to diverge from linear behavior. The non-linear behavior is attributed to multiple factors, mainly heat and polarization shifting at high voltages. The coupling coefficients dictate the mechanical deformation of the composite under pure electric loading in the direction of the fibers. In analogy, the composite stiffness matrix dictates the mechanical deformation of the composite under pure mechanical loading.

In the macro-scale boundary conditions such as mechanical constraints significantly affect the structural response, thus the values computed through CUF diverge slightly from the TFA as shown in In Fig. 5. Similar to TFA during the initial part of the curve the FE model match well with experimental results accordingly this model presents an adequate approximation of the structural behavior and can be extended to evaluate the behavior of the structure in damaged conditions.

The voltage increase in the damaged model is expected due to the stiffness drop of the entire structure. The amplitude of the voltage fluctuates depending on the location of the void along the length. The global change in voltage signifies clear indication of damage occurrence in the structure. Localization through local voltage drops in the vicinity of the void is evaluated. Figure 7 demonstrates the voltage variation in the proximity of the void; with the void being located at different locations relative to the $\mathrm{x}, \mathrm{y}$ coordinates. The local voltage difference descends the further the location of the applied load from the damaged area. However, the sensitivity also diminishes; indicating the significance of the distance between void location and applied load in damage localization.

\section{CONCLUSION}

In the current study a multi-scale approach been utilized to extract the behavior of composite structures with electrically active fibers. The model demonstrated proper approximation of the experimental results. Both models, the micro-scale (TFA) model and macro-scale (CUF-FEM) model, been successfully utilized through a hierarchal approach; accuracy is increased substantially with fiber-fiber interaction scheme of the TFA in micro-scale and the structural response in macro-scale. Extracting the electro-mechanical properties of the composite material from the micro-mechanical model allows a significant reduction in the computational cost at the macro-scale and 
therefore enables the modeling of more complex geometry and structures utilizing the proposed multi-scale approach.

Furthermore, damage had been introduced and evaluated in a macro-scale finite element analysis through introducing voids at different locations of the model. Damage identification and localization is done through analyzing the impact of the damage. Global voltage variation along the entire model provides clear indication of damage occurrence, while localized fluctuation of voltage near the vicinity of the damage due to different void's location demonstrated a dependence on the distance from the applied load and boundary condition. With sufficient database of results the work presented in this paper can be further expanded to include damage characterization through proper implementation of Genetic Algorithms [GA] and Neural Networks [NN] [23].

\section{REFERENCES}

[1] Chopra, Inderjit. Review of State of Art of Smart Structures and Integrated Systems. AIAA, 2002, 40, 2145-2187.

[2] Crawley, Edward and de Luis, Javier. Use of Piezoelectric actuators as elements of intelligent structures. AIAA,1987, 25, 1373-1385.

[3] Victor Giurgiutiu and Andrei N. Zagrai. Embedded Self Sensing Piezoelectric Active Sensors for On-line Structural identication. Vibration and Acoustics, 2002, 124, 116-125.

[4] Zagrai, Andrei and Giurgiutiu, Victor. Electro-Mechancial Impedance Method for Crack Detection in Thin Plates. Intelligient Materials systems and Structures, 2001, 12, 709-718.

[5] Giurgiutiu, Victor and Rogers, Craig. Recent Advancements in the ElectroMechanical (E/M) Impedance Method for Structural Health Monitoring and NDE.

Annual International Symposium on Smart Structures and Materials, 1998, 3329-3353.

[6] Smith, W. A. and Auld, B. A. Modeling 1-3 Composite Plezoelectrics: ThicknessMode Oscillations. Ultrasonics, Feroelectrics, and Frequency Control, 1991, 38, 40-47.

[7] Bent, A. A.; Hagood, N. W.; Rodgers, J. P. Anisotropic Actuation with Piezoelectric Fiber Composites. Intelligent Material Systems and Structures, 1995, 6, 338-349.

[8] Williams, R. B.;Park, Gyuhae;Inman, D. J. and Wilkie, W. K. An overview of Composite Acutators with Piezoceramics. Conference on Structural Dynamics, Westin Los Angeles Airport Hotel,Los Angeles, CA, February 4th -7th, 2002. 421-427.

[9] Eshelby, J. D. The determination of the elastic field of an ellipsoidal inclusion, and related problems.Proceedings of Royal Society of London, 1957, A241, 376-396.

[10] Dunn, M. L. and Taya, M. Micromechanics Predictions of the Effective Electroelastic Moduli of Piezoelectric Composites. International Journal of Solids and Structures, 1993, 443, 161-175.

[11] Mori, T and Tanaka, K. Average stress in matrix and average elastic energy of materials with misfitting inclusions. Acta Metallurgica, 1973, 21, 571-574. 
[12] Li, Jiang Yu and Dunn, Martin L. Micromechanics of Magnetoelectroelastic Composite Materials: Average Fields and Effective Behavior. Intelligent Material Systems and Structures, 1998, 9, 404-416.

[13] Berger, H.;Kari, S.;Gabbert, U.; Rodriguez-Ramos, R.; Guinovart, R.; Otero, J. A. and Castillero, J. B.An analytical and numerical approach for calculating effective material coefficients of piezoelectric fiber composites. International Journal of Solids and Structures, 2005, Vol. 42, 5692-5714.

[14] Bahei-El-Din, Y. A. Modelling electromechanical coupling in woven composites exhibiting damage. Proceedings of IMechE 223, Part G: J Aerospace Engineering, 2009, 485-495.

[15] Carrera, E.Theories and Finite Elements for Multilayered Plates and Shells:A Unified compact formulation with numerical assessment and benchmarking., Archives of Computational Methods in Engineering, 2003, 10, 215-296.

[16] Carrera, Erasmo. Plates and Shells for Smart Structures: Classical and Advanced Theories for Modelling and Analysis. Torino : Wiley, 2008.

[17] Dvorak, G. J. Transformation field analysis of inelastic composite materials. Proceeding of Royal Society of London, 1992, A437, 311-327.

[18] IEEE, Std 176. Institute of Electrical and Electronics Engineers Standard on Piezoelectricity : ANSI/IEEE, 1987.

[19] Ikeda, T. Fundamentals of Piezoelectricity : Oxford University Press, 1996.

[20] Teply, J. L. Periodic Hexagonal Array Models for Plasticity Analysis of Composite Materials. Ph. D. Dissertation: University of Utah, 1984.

[21] Advanced Cerametrics Incorporated. Piezoelectric Fiber Composites.

[22] Berlincourt, D; Krueger, H. H. A. and Near, C. Technical Publications 226: mportant Properties of Morgan Electro Ceramics Piezoelectric Ceramics (PZT4, PZT-5A, PZT-5H, PZT-8) : Morgan Electro Ceramics, 2013.

[23] Hatem, T. M.; Abuelfoutouh, M. N.; Negm, H. M. Application of Genetic Algorithms and Neural Networks to Health Monitoring of Composite Structures. Proceedings of the Second European Workshop on Structural Health Monitoring, Munich, Germany, July 7-9, 2004, 616-632; Boller, C., Staszewski, W. J.; DEStech Publications Inc, 2004.

\section{TABLE AND FIGURES}

Table 1. Material Properties of PZT-5A as extracted from [22,14].

\begin{tabular}{|c|c|c|c|c|c|c|}
\hline Material & $\boldsymbol{E}_{L}(G P a)$ & $v_{L}$ & $d_{33}$ & $\begin{array}{r}\boldsymbol{d}_{31} \\
x 10^{-}\end{array}$ & $d_{15}$ & $\begin{array}{c}\varepsilon_{33} \\
\left(\frac{C}{V \cdot m} \times 10^{-9}\right)\end{array}$ \\
\hline PZT 5-A & 69 & 0.34 & 374 & 171 & 584 & 15000 \\
\hline Epoxy & 3.35 & 0.35 & - & - & - & - \\
\hline
\end{tabular}




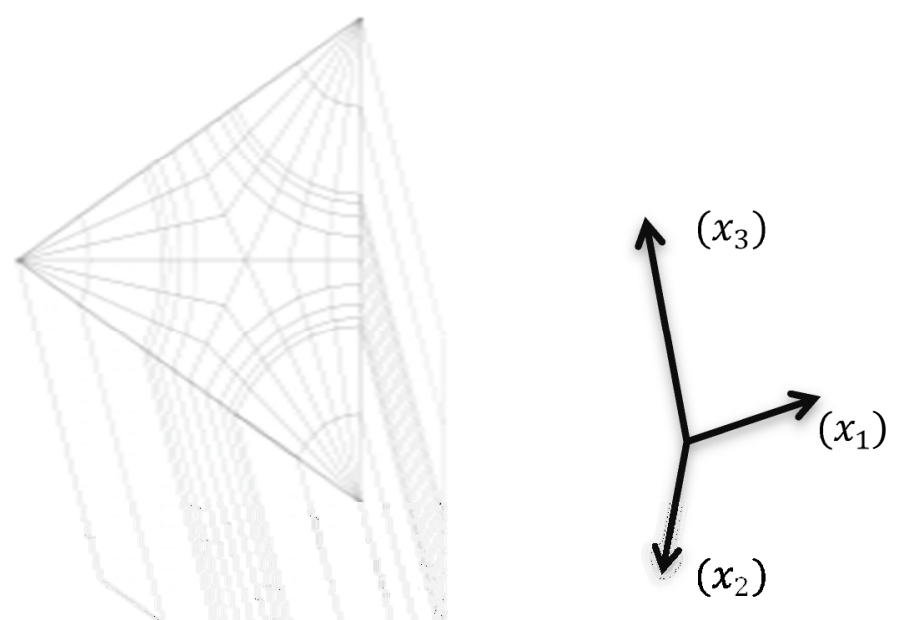

Fig. 1. PHA Representative volume element.

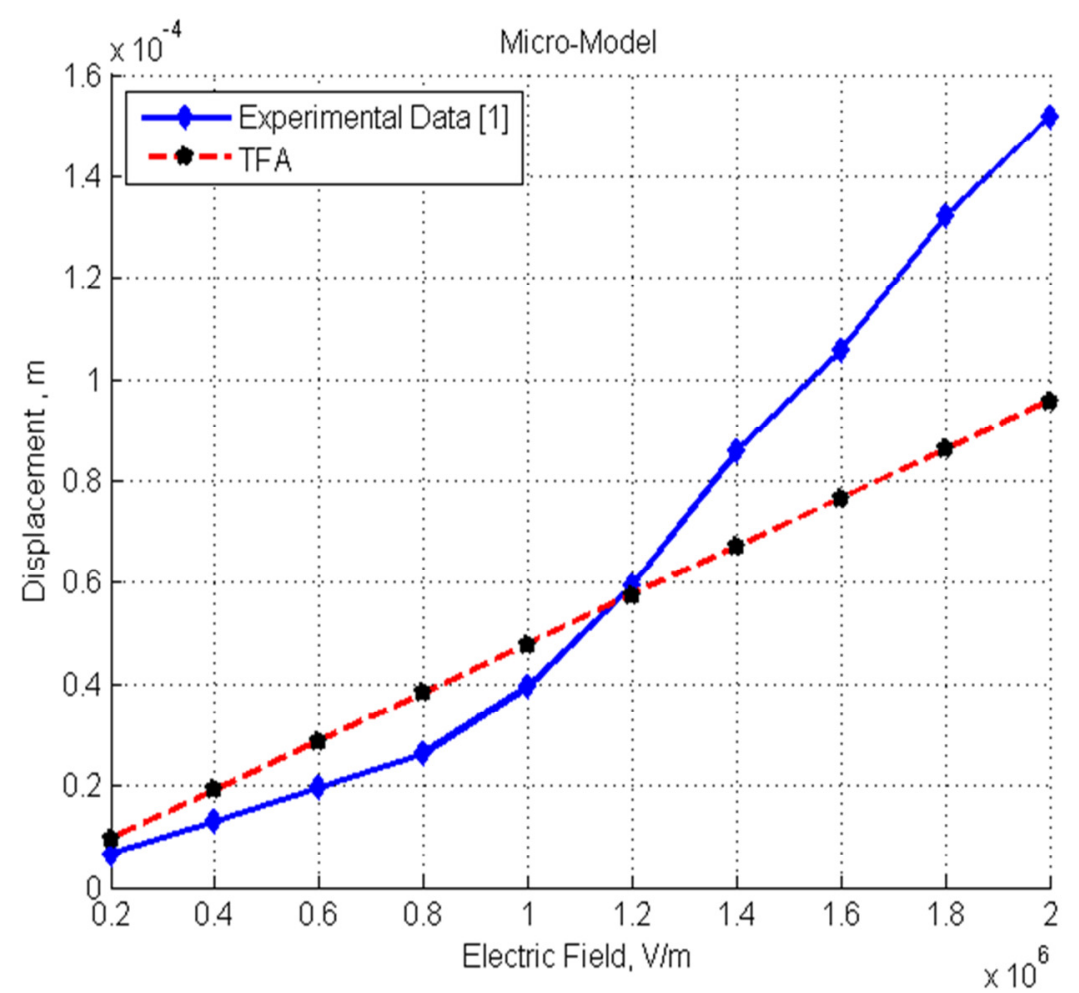

Fig. 2. Experimental data compared with TFA results for mechanical deformation under applied electrical load ${ }^{1}$

${ }^{1}$ Experimental data are obtained from Advanced Ceramics Inc. data sheet for PFC14 specimen 


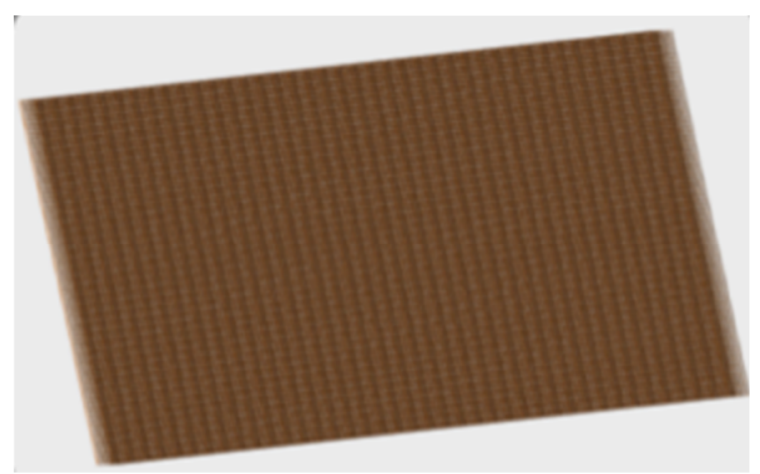

(a)

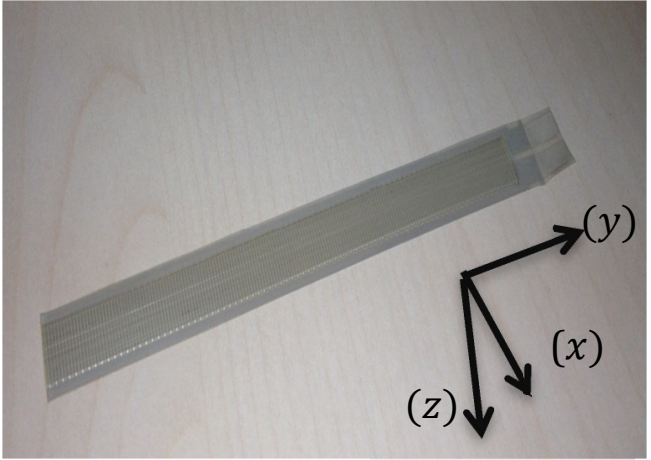

(b)

Fig. 3. PFC composite: (a) Finite element mesh, (b) PFC composite provided by Advanced Ceramics Incorporated.

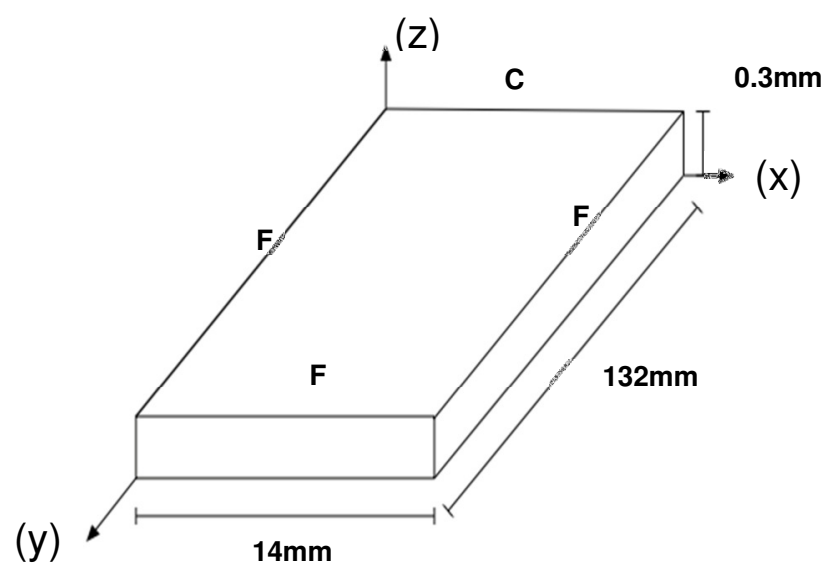

(a)

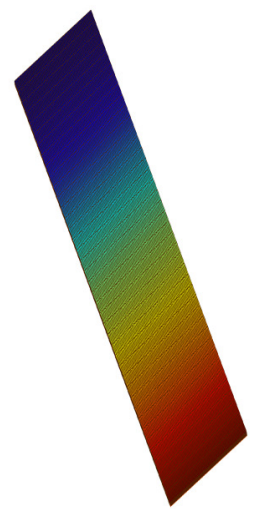

(b)

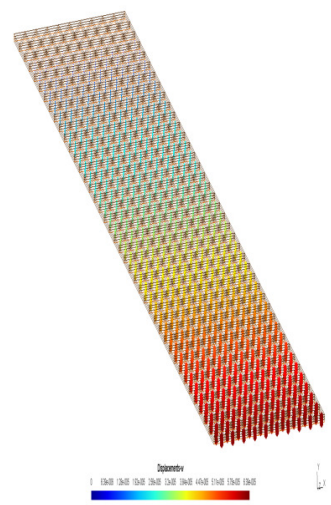

(c)

Fig. 4. CUF solution for electrical actuation model (a) Geometry, dimensions and boundary conditions of the numerical model [C indicates clamped side while $F$ is free], (b) Voltage distribution along length grounded at blue and applied at red, (c) Displacement distributions induced by electro-mechanical coupling displayed in Fig. 5. 


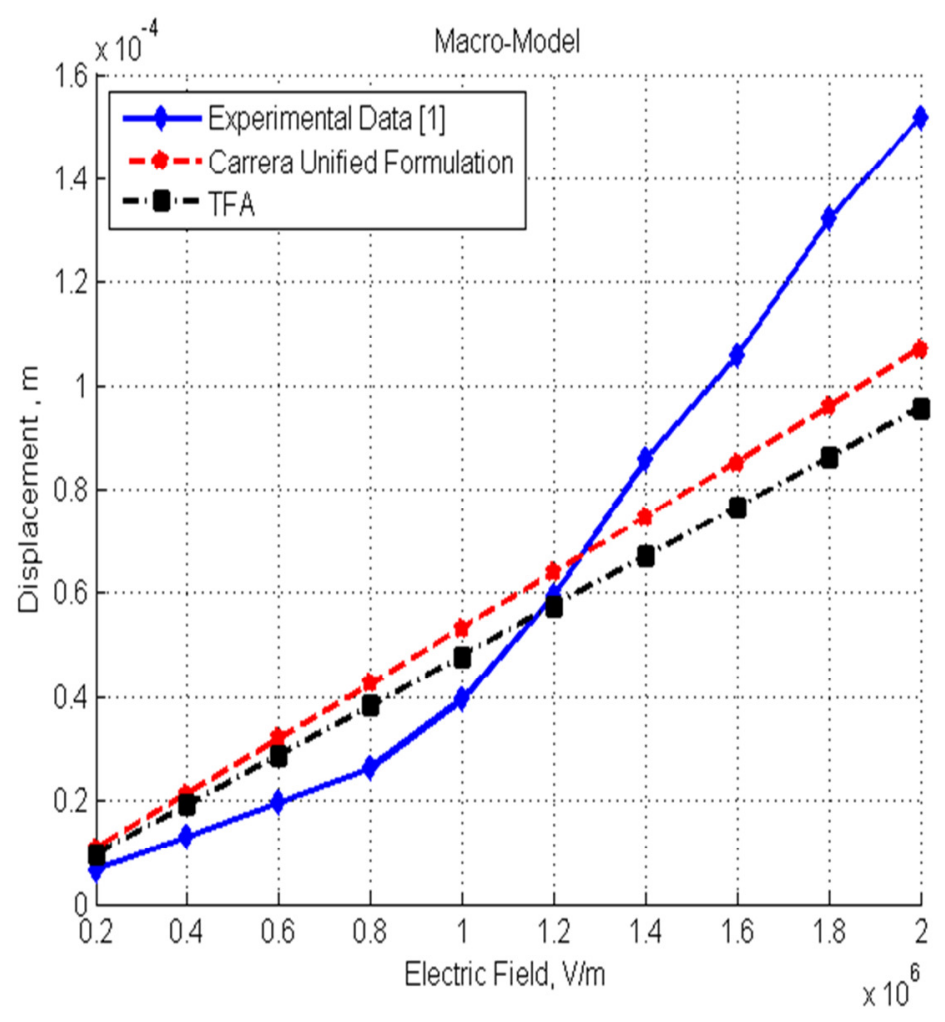

Fig. 5. Displacement against Electrical Field solved using Carrera Unified Formulation and compared to the experimental and TFA results.

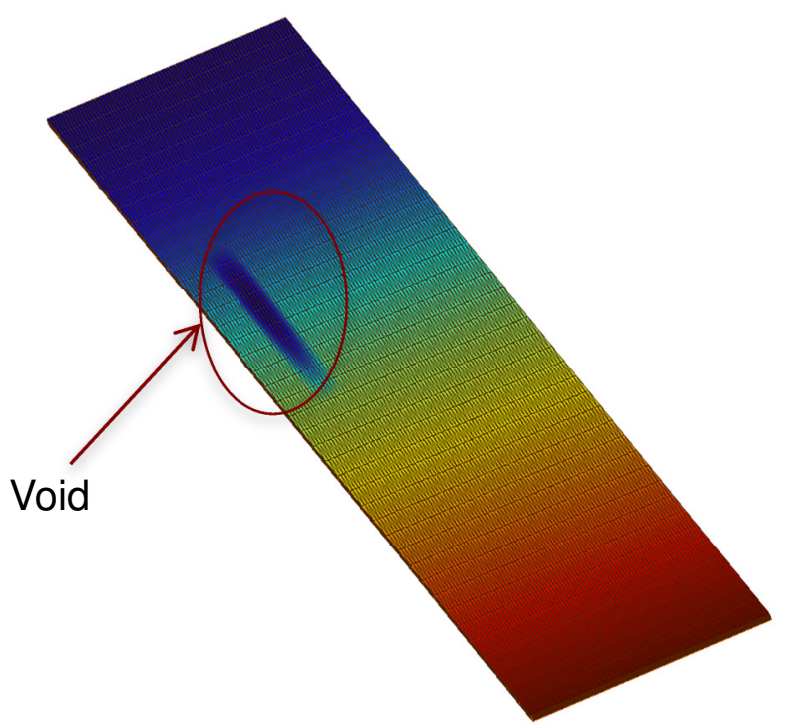

Fig. 6.Voltage distribution in a damaged model with introduced void. 


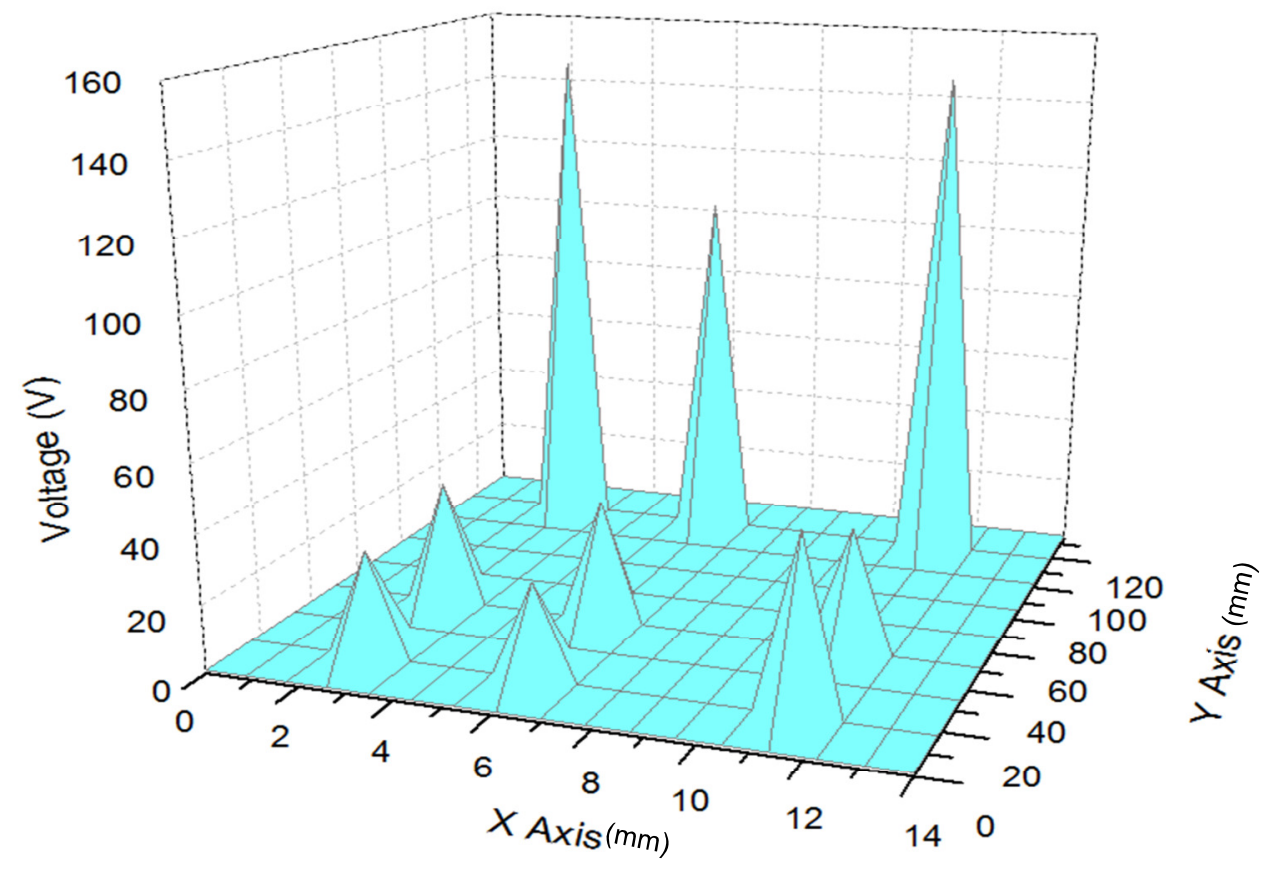

Fig. 7. Voltage variation in proximity of void for varying damaged locations along the length of the model. 